\title{
The two faces of the twisted Kummer surface
}

\author{
by
}

\author{
ADAM LOGAN (Montréal, QC)
}

1. Introduction. Let $C$ be a curve of genus 2 defined by an equation of the form $y^{2}=f(x)$ over a number field $F$. In studying the arithmetic of $C$, it is necessary to consider the $\operatorname{Jacobian} \operatorname{Jac}(C)$ of $C$, an abelian variety of dimension 2. However, this variety is rather difficult to compute with directly (it is most naturally embedded into projective space as the intersection of 72 quadrics in $\left.\mathbb{P}^{15}\right)$. Accordingly, one often considers the quotient of $\operatorname{Jac}(C)$ by the involution -1 that takes the class of a divisor $D$ to the class of $-D$. This quotient, the Kummer surface of $C$, can be embedded as a quartic surface in $\mathbb{P}^{3}$ with 16 nodes; this is the largest number of isolated singularities possible for a quartic surface in $\mathbb{P}^{3}$. The minimal desingularization of the quotient can also be embedded as the intersection of three quadrics in $\mathbb{P}^{5}$.

To determine the rank of the Jacobian over $F$ by the method of 2descent, it is necessary to study certain twists of $\operatorname{Jac}(C)$ - these are varieties isomorphic to $\operatorname{Jac}(C)$ over $\bar{F}$ (the algebraic closure of $F$ ), but not necessarily over $F$ itself. These are twists by Galois cocycles with values in the set of translations by 2-torsion points. Since translation by a 2-torsion point commutes with multiplication by -1 on $\operatorname{Jac}(C)$, the same cocycles define twists of $\operatorname{Jac}(C) / \pm 1$ (or equivalently, multiplication by -1 is still defined on the twists; the quotient of the twist is isomorphic to the twist of the quotient). The minimal desingularizations of these twists of $\operatorname{Jac}(C) / \pm 1$ can again be embedded as the intersection of three quadrics in $\mathbb{P}^{5}$. In previous work [7, Ronald van Luijk and I studied the geometry and arithmetic of these twists using this embedding: we determined the Néron-Severi group in the generic case, studied the configuration of lines, analyzed some elliptic fibrations, and proved that some of these surfaces have no rational points by means of the Brauer-Manin obstruction.

2010 Mathematics Subject Classification: Primary 14J28; Secondary 11G30, 14G05.

Key words and phrases: Kummer surface, Brauer-Manin obstruction. 
In this paper, we study the twists of $\operatorname{Jac}(C) / \pm 1$ further, considering not only the embedding in $\mathbb{P}^{5}$ but also the map to $\mathbb{P}^{3}$ and the quartic surface which is its image. The main arithmetical result follows:

Theorem 1.1. Let $f=x^{6}-3 x^{5}+3 x^{4}-3 x^{2}-x-1$, let $A_{f}=\mathbb{Q}[x] /(f(x))$, let $C$ be the curve $y^{2}=f(x)$, and let $\delta \in A_{f}$ be the image of $x^{5}-7 x^{4}+4 x^{3}+$ $3 x^{2}+x$. Then the Brauer-Manin obstruction blocks the existence of rational points on $V_{f, \delta}$, a twist of the Kummer surface of $C$ defined in Section 3. The minimal desingularization of $V_{f, \delta}$ is defined by the polynomials

$$
\begin{aligned}
3 x_{1}^{2} & +3 x_{1} x_{2}-4 x_{1} x_{3}-19 x_{1} x_{4}-26 x_{1} x_{5}-7 x_{1} x_{6}-2 x_{2}^{2}-19 x_{2} x_{3} \\
& -26 x_{2} x_{4}-7 x_{2} x_{5}+54 x_{2} x_{6}-13 x_{3}^{2}-7 x_{3} x_{4}+54 x_{3} x_{5}+125 x_{3} x_{6} \\
& +27 x_{4}^{2}+125 x_{4} x_{5}+112 x_{4} x_{6}+56 x_{5}^{2}-105 x_{5} x_{6}-261 x_{6}^{2}, \\
x_{1}^{2} & -8 x_{1} x_{3}-8 x_{1} x_{4}+10 x_{1} x_{5}+62 x_{1} x_{6}-4 x_{2}^{2}-8 x_{2} x_{3}+10 x_{2} x_{4} \\
& +62 x_{2} x_{5}+134 x_{2} x_{6}+5 x_{3}^{2}+62 x_{3} x_{4}+134 x_{3} x_{5}+184 x_{3} x_{6} \\
& +67 x_{4}^{2}+184 x_{4} x_{5}+164 x_{4} x_{6}+82 x_{5}^{2}+128 x_{5} x_{6}+183 x_{6}^{2}, \\
5 x_{1}^{2} & +17 x_{1} x_{2}+34 x_{1} x_{3}+3 x_{1} x_{4}-74 x_{1} x_{5}-191 x_{1} x_{6}+17 x_{2}^{2}+3 x_{2} x_{3} \\
& -74 x_{2} x_{4}-191 x_{2} x_{5}-222 x_{2} x_{6}-37 x_{3}^{2}-191 x_{3} x_{4}-222 x_{3} x_{5} \\
& -33 x_{3} x_{6}-111 x_{4}^{2}-33 x_{4} x_{5}+382 x_{4} x_{6}+191 x_{5}^{2}+601 x_{5} x_{6}-137 x_{6}^{2} .
\end{aligned}
$$

The results of this article do not have any direct relevance to 2-descent, because the element of the Brauer group used is nontrivial only for quotients of twists of the Jacobian that are known not to have rational points. On the other hand, we give an example of a Brauer-Manin obstruction in an arithmetically "generic" situation in which the degree of the field of definition of generators of the Néron-Severi group is as large as possible. This is quite unusual and interesting.

In addition, I expect that this advance in understanding the divisors and linear systems on twists of $\operatorname{Jac}(C) / \pm 1$ will allow further constructions of Brauer-Manin obstructions using other elements of the Brauer group, including some that are important for 2-descent. This would allow constructing curves of genus 2 of which infinitely many twists have nontrivial Tate-Shafarevich group, as in [7, Theorem 1.1].

It is likely that there are infinitely many $\delta_{i} \in A_{f}$ that are distinct in $A_{f}^{*} /\left(\left(A_{f}^{*}\right)^{2} \mathbb{Q}^{*}\right)$ for which the Brauer-Manin obstruction blocks the existence of rational points on the twist of $\operatorname{Jac}(C) / \pm 1$ by $\delta_{i}$. This question is discussed in Remark 6.4 at the end of the paper.

2. Quadratic forms. In this section we discuss some general properties of quadratic forms and quadric hypersurfaces. Our standing convention will be that all such forms and hypersurfaces are nonsingular. 
We begin with the well-known classification of quadratic forms over a number field. Since every quadratic form may be diagonalized by a linear change of coordinates, it suffices to consider only diagonal quadratic forms.

Definition 2.1. Let $F$ be a number field, let $\mathfrak{p}$ be a place of $F$, and let $a_{1}, \ldots, a_{n}$ be a sequence of elements of $F$ or $F_{\mathfrak{p}}$. The Hasse symbol $\left(a_{1}, \ldots, a_{n}\right)_{\mathfrak{p}}$ is the product of Hilbert symbols $\prod_{i \leq j}\left(a_{i}, a_{j}\right)_{\mathfrak{p}}$.

Proposition 2.2 ([9, Theorem 63.20]). Diagonal quadratic forms $\sum_{i=1}^{n} a_{i} x_{i}^{2}$ over a local field $F_{\mathfrak{p}}$ are classified by the following invariants: $n, \prod a_{i}$ up to squares, the Hasse symbol $\left(a_{1}, \ldots, a_{n}\right)_{\mathfrak{p}}$, and, if $\mathfrak{p}$ is a real place, the number of positive $a_{i}$.

Proposition 2.3 ([9, Theorem 66.4, Remark 66.5]). Over a global field $F$, diagonal quadratic forms $\sum_{i=1}^{n} a_{i} x_{i}^{2}$ are classified by the invariants $n, \prod a_{i}$ up to squares, the $\left(a_{1}, \ldots, a_{n}\right)_{\mathfrak{p}}$ for all nonarchimedean $\mathfrak{p}$, and, for each real place $\mathfrak{p}_{\infty, j}$, the number of $a_{i}$ that are positive at $\mathfrak{p}_{\infty, j}$. The product of the $\left(a_{1}, \ldots, a_{n}\right)_{\mathfrak{p}}$ is 1 .

One distinction between quadratic forms and quadric hypersurfaces is that the isomorphism class of the first may be affected by scaling, whereas that of the second is not. We show, however, that this problem does not arise in an important special case.

Proposition 2.4. Let $n$ be even and let $f\left(x_{1}, \ldots, x_{n}\right)$ be a quadratic form over a nonreal local field $F_{\mathfrak{p}}$ with determinant $(-1)^{n / 2}$ up to squares. Then $f$ is equivalent to $c f$ for all $c \neq 0 \in F_{\mathfrak{p}}$.

Proof. We may suppose $f$ to be a diagonal form $\sum_{i} a_{i} x_{i}^{2}$; the determinant is then $\prod_{i} a_{i}$. Since $n$ is even, the determinant is the same up to squares for $f$ and $c f$, so it suffices to show that $\left(a_{1}, \ldots, a_{n}\right)_{\mathfrak{p}}=\left(c a_{1}, \ldots, c a_{n}\right)_{\mathfrak{p}}$. Expanding this out, we have

$$
\begin{aligned}
\left(c a_{1}, \ldots, c a_{n}\right)_{\mathfrak{p}} & =\prod_{i \leq j}\left(c a_{i}, c a_{j}\right)_{\mathfrak{p}}=\prod\left(a_{i}, a_{j}\right)_{\mathfrak{p}}\left(c, a_{j}\right)_{\mathfrak{p}}\left(a_{i}, c\right)_{\mathfrak{p}}(c, c)_{\mathfrak{p}} \\
& =\left(a_{1}, \ldots, a_{n}\right)_{\mathfrak{p}} \prod_{i}\left(\left(c, a_{i}\right)_{\mathfrak{p}}^{n+1}(c, c)_{\mathfrak{p}}^{n(n+1) / 2}\right) \\
& =\left(a_{1}, \ldots, a_{n}\right)_{\mathfrak{p}}\left(c, \prod a_{i}\right)_{\mathfrak{p}}(c, c)_{\mathfrak{p}}^{n(n+1) / 2} .
\end{aligned}
$$

If $4 \mid n$, then $\prod a_{i}$ is a square, so $\left(c, \prod a_{i}\right)_{\mathfrak{p}}=1$, and $n(n+1) / 2$ is even, so $(c, c)_{\mathfrak{p}}^{n(n+1) / 2}=1$. If $n \equiv 2(\bmod 4)$, then $-\prod a_{i}$ is a square, so $\left(c, \prod a_{i}\right)_{\mathfrak{p}}=$ $(c,-1)_{\mathfrak{p}}$, and $(c, c)_{\mathfrak{p}}^{n(n+1) / 2}=(c, c)_{\mathfrak{p}}$, so the product of the last two factors is $(c,-c)_{\mathfrak{p}}=1$.

COROLlary 2.5. Let $n$ be even and let $f\left(x_{1}, \ldots, x_{n}\right)$ be a quadratic form over a global field $F$ with determinant $(-1)^{n / 2}$ up to squares. Then $f$ is 
equivalent to cf for all $c \neq 0 \in F$ that are positive at every real place at which the signature of $f$ is nonzero.

Proof. This follows by combining Proposition 2.3 with Proposition 2.4 .

REMARK 2.6. Let $F$ be a global field. Then we may associate an element of $\operatorname{Br}_{2}(F)$ to every quadric hypersurface in $\mathbb{P}^{n-1}$ defined by an equation with determinant $(-1)^{n / 2}$ up to squares: namely, we diagonalize an equation defining the hypersurface to obtain $\sum_{i=1}^{n} a_{i} x_{i}^{2}$ and choose the element whose local invariant at $\mathfrak{p}$ is $1 / 2$ at exactly those places where $\left(a_{1}, \ldots, a_{n}\right)_{\mathfrak{p}}=-1$. Proposition 2.4 shows that this element does not depend on the choice of defining equation.

Now we discuss varieties. First we recall some results on linear subspaces of quadric hypersurfaces over an algebraically closed field. Following [10, Definition 1.1], we define a generator of a quadric hypersurface of dimension $k$ to be a linear subspace of dimension $\lfloor k / 2\rfloor$.

Proposition 2.7 ([10, Theorem 1.2, Remark 1.4(ii), Lemma 1.5]). Let $Q$ be a smooth quadric hypersurface of even dimension $2 l$ over an algebraically closed field $\bar{F}$ of characteristic not 2 . On $Q$, the variety of generators (viewed as a subvariety of the Grassmannian, for example) has two irreducible components, each nonsingular of dimension $\left(\begin{array}{c}l+1 \\ 2\end{array}\right)$. Every linear subspace of dimension $l-1$ is contained in exactly one generator from each component.

In the case $l=2$, we may describe the families of lines and planes concretely when $Q$ is the Grassmannian $G$ of lines in $\mathbb{P}^{3}$ in its Plücker embedding. (We will take $G$ to be the quadric hypersurface in $\mathbb{P}^{5}$ defined by the polynomial $Q_{g}=x_{0} x_{5}-x_{1} x_{4}+x_{2} x_{3}$. Readers who prefer sign and order conventions for minors other than those implied by this equation may make the few changes that will be necessary.) In this case, [3, Section 17.2] states that every line in the 5-dimensional family of lines on $G$ parametrizes a family of lines in $\mathbb{P}^{3}$ that contains a fixed point and is contained in a given plane. One of the two families of planes parametrizes the lines in $\mathbb{P}^{3}$ that contain a fixed point; the other, those lying in a fixed plane.

For completeness, we sketch proofs of these well-known results. In light of Proposition 2.7 above, to prove the statement on the families of planes it suffices to verify that the lines containing $[0: 0: 0: 1]$ and those contained in the plane $y_{0}=0$ constitute planes. For the first, the three Plücker coordinates not involving $y_{3}$ are 0 , so we get a plane; for the second, the three involving $y_{0}$ are 0 , and again we obtain a plane. Clearly, the same statements will hold for any point and any plane in $\mathbb{P}^{3}$. But then a line in $G$ is the intersection of the two planes containing it, so the description of lines that we have given is evident. 
Definition 2.8. For a point $P$ and a plane $H$ in $\mathbb{P}^{3}$ such that $P$ lies on $H$, let $l_{P, H}$ be the family of lines in $\mathbb{P}^{3}$ that contain $P$ and are contained in $H$. (From the above, these lines determine a line in $G$.) Let $\pi_{1}$ be the map from the variety of lines on $G$ to $\mathbb{P}^{3}$ that takes $l_{P, H}$ to $P$, and let $\pi_{2}$ be the map from the variety of lines on $G$ to the dual $\mathbb{P}^{3}$ that takes $l_{P, H}$ to $H$.

LEMMA 2.9. Let $Q$ be a smooth quadric hypersurface in $\mathbb{P}^{2 n-1}$ over an algebraically closed field $\bar{F}$ of characteristic not equal to 2 . Then the family of $n-2$-planes on $Q$ is irreducible.

Proof. Let $A$ be this family and let $\check{\mathbb{P}}^{2 n-1}$ be the dual of $\mathbb{P}^{2 n-1}$. Consider the incidence correspondence $I \subset A \times \check{\mathbb{P}}^{2 n-1}$ consisting of $n-2$-planes lying in the hyperplane. Let $U$ be the open subset of $I$ lying above points of $\check{\mathbb{P}}^{2 n-1}$ corresponding to hyperplanes whose intersection with $Q$ is smooth. On $U$, the fibers of the projection to the second factor are irreducible ([10, Theorem 1.2(a)]); since the image of the projection is an open subset of $\check{\mathbb{P}}^{2 n-1}$, hence irreducible, it follows that $U$ is irreducible. So it suffices to show that $I$ is the closure of $U$.

Choose a point $(O, H)$ not in $U$, so that $H \cap Q$ is singular and $O$ is an $n-2$-plane in $H \cap Q$. As in [10, Lemma 1.3(b)], choose coordinates so that $Q$ is defined by $\sum_{i=1}^{n} x_{i} y_{i}=0$ and $O$ is defined by $x_{1}=\cdots=x_{n}=y_{1}=0$. Then $H$ is defined by $\sum c_{i} x_{i}+d_{1} y_{1}=0$. For $H \cap Q$ to be singular is equivalent to $c_{1}=0$ or $d_{1}=0$. Clearly, in either case there is an open curve in $U$ lying above $O$ whose closure contains the point $(O, H)$, which shows that $(O, H)$ in fact lies in $\bar{U}$. It follows that $I$ is irreducible and hence that $A$ is irreducible.

We will use this lemma and the next two propositions to understand the generators of a quadric hypersurface of even dimension over a general field. Suppose that $Q$ is defined over a smaller field $F$, and let us consider the generators of $Q$ over $F$.

Proposition 2.10. Let $Q$ be a quadric hypersurface over $F$ in $\mathbb{P}^{2 n-1}$. Then $Q$ contains an F-rational n-1-plane if and only if $Q$ is $F$-isomorphic to the variety defined by $\sum_{i=1}^{n} x_{i} x_{n+i}=0$.

Proof. If $Q$ contains an $F$-rational $n$ - 1 -plane $P$, we may suppose that $P$ is defined by the vanishing of the linear forms $l_{1}, \ldots, l_{n}$. We may write the equation of $Q$ as $\sum_{i=1}^{n} l_{i} m_{i}$, where the $m_{i}$ are linear forms (not uniquely). If the $l_{i}$ and $m_{i}$ all vanished at a common point, then $Q$ would be singular there; hence the $l_{i}$ and $m_{i}$ constitute a basis of $\mathcal{O}_{\mathbb{P}^{n}}(1)$, and we may change coordinates by putting $x_{i}=l_{i}, x_{n+i}=m_{i}$. The converse is easy.

Proposition 2.11. The field of definition of the families of generators of $Q$ is $F\left(\sqrt{(-1)^{n} \operatorname{det} M}\right)$, where $M$ is the matrix corresponding to $Q$. 
REMARK 2.12. The proof that follows is modeled on that of [2, Lemma 2.5] for the case $n=2$, and is included for lack of a suitable reference (the statement is well-known). We will only use the result in the case $n=3$, in which it is proved in [3, Lemma 17.1.1].

Proof of Proposition 2.11. First, the result is easy to prove for $n=1$ : it says that the polynomial $a x^{2}+2 b x y+c y^{2}$ factors over $F\left(\sqrt{b^{2}-a c}\right)$. In general, since there are two families of generators, the field of definition will be at worst a quadratic extension of $F$. We will denote this extension by $F(\sqrt{g})$ (allowing the possibility that $g$ is a square if the families are already defined over $F$ ).

Let $F\left(A_{n-2}\right)$ be the function field of the Fano scheme of $n$-2-planes on $Q$. Suppose that we can show that the field of definition of the families of generators over $F\left(A_{n-2}\right)$ is $F\left(A_{n-2}\right)\left(\sqrt{(-1)^{n} \operatorname{det} M}\right)$. Since $F\left(A_{n-2}\right)(\sqrt{g})$ is also the field of definition of the families of generators over $F\left(A_{n-2}\right)$, it is isomorphic to $F\left(A_{n-2}\right)\left(\sqrt{(-1)^{n} \operatorname{det} M}\right)$. We just showed in Lemma 2.9 that $F\left(A_{n-2}\right)$ is geometrically integral, so $F$ is algebraically closed in $F\left(A_{n-2}\right)$. It follows that $F(\sqrt{g}) \cong F\left(\sqrt{(-1)^{n} \operatorname{det} M}\right)$.

Thus we may assume that there is an $n-2$-plane on $Q$ defined over $F$ (since there is certainly one defined over $F\left(A_{n-2}\right)$ ). Let $G_{1}, G_{2}$ be the generators containing $O$, and $\mathcal{F}_{1}, \mathcal{F}_{2}$ the $\bar{F}$-irreducible components of the variety of generators. If $G_{1}$ is defined over some field $L$, then so is $\mathcal{F}_{1}$, for otherwise some element of $\operatorname{Gal}(\bar{L} / L)$ would take $\mathcal{F}_{1}$ to $\mathcal{F}_{2}$ while fixing the point corresponding to $G_{1}$. This is impossible, since $\mathcal{F}_{1} \cap \mathcal{F}_{2}$ is empty. It therefore suffices to show that the field of definition of $G_{1}$ and $G_{2}$ is $F\left(\sqrt{(-1)^{n} \operatorname{det} M}\right)$.

Let $l_{1}, \ldots, l_{n+1}$ be a basis for the linear forms vanishing on $O$, and extend them to a basis of $\mathcal{O}(1)$ with forms $m_{1}, \ldots, m_{n-1}$. Since $Q$ contains $O$, its defining equation is of the form $\sum_{i=1}^{n-1} g_{i}\left(l_{1}, \ldots, l_{n+1}\right) m_{i}+q\left(l_{1}, \ldots, l_{n+1}\right)$, where the $g_{i}$ are linear and $q$ is quadratic. An $n-1$-plane containing $O$ contains a point $\left(a_{1}, \ldots, a_{n+1}, b_{1}, \ldots, b_{n-1}\right)$ with not all $a_{i}=0$; for the $n-1$-plane spanned by such a point and $O$ to lie on $Q$ is equivalent to $g_{i}\left(a_{1}, \ldots, a_{n+1}\right)=0$ for all $i$ and $q\left(a_{1}, \ldots, a_{n+1}\right)=0$ and so depends only on the $a_{i}$.

The $g_{i}$ must be linearly independent, because $Q$ is singular on their common zero locus. Hence we may change coordinates so as to replace $l_{1}, \ldots, l_{n+1}$ by $g_{1}, \ldots, g_{n-1}$ followed by two of the $l_{i}$. We have only made linear changes of coordinates, so the class of the determinant in $F^{*} /\left(F^{*}\right)^{2}$ has not changed. On the other hand, in these new coordinates the first $n-1$ coordinates of the points we are looking for are 0 , so by the case $n=1$ the field of definition of the two points is $F(\sqrt{-D})$, where $D$ is the determinant of the matrix obtained from that of $Q$ in the new coordinates by deleting all columns and rows except those corresponding to the two $l_{i}$. 
Calculating the determinant of the matrix of $Q$ in this new basis by expanding $n-1$ times alternately along the top row and left column, we see that its determinant is equal to $(-1)^{n-1} D$, so the field of definition is $F\left(\sqrt{(-1)^{n} \operatorname{det} M}\right)$, where $M$ is the matrix of $Q$.

Suppose further that $n=3$, so that $\operatorname{dim} Q=4$, and that $F$ is a local field or a global field. In this case, we have three elements of $\operatorname{Br}_{2}(F)$ : that defined by the local invariants of $Q$ (which we call $b_{1}$ ) and those classifying the Severi-Brauer varieties which are the bases of the families of planes (denoted $\left.b_{2}, b_{3}\right)$. These are of order 2 because they are trivialized by a quadratic extension in which all primes where $Q$ is not isomorphic to $Q_{g}$ are ramified or inert.

Proposition 2.13. The three elements $b_{1}, b_{2}, b_{3}$ of $\mathrm{Br}_{2}(F)$ defined above are all equal.

Proof. Observe that $Q_{g}=x_{0} x_{5}-x_{1} x_{4}+x_{2} x_{3}$ is isomorphic to $x_{0}^{2}+x_{1}^{2}+$ $x_{2}^{2}-x_{3}^{2}-x_{4}^{2}-x_{5}^{2}$ and hence that its local invariants are all 1 . Therefore, the element $b_{1}$ is nontrivial exactly at those places where $Q$ is not isomorphic to $Q_{g}$; which, by Proposition 2.10, are exactly the places at which the two families of planes on $Q$ do not have a rational point, which are the places where $b_{2}$ and $b_{3}$ are nontrivial.

It can still be proved that $b_{2}=b_{3}$ for an arbitrary field $F$ of characteristic not equal to 2 , but I see no way to define an analogue of $b_{1}$, nor any reason why $b_{2}, b_{3}$ should belong to $\operatorname{Br}_{2}(F)$ (a priori they only belong to $\mathrm{Br}_{4}(F)$ ).

3. The Kummer surface. The purpose of this section is to describe the embedding of the twisted Kummer surface by three quadrics in $\mathbb{P}^{5}$. For more details the reader is referred to [7, Section 2].

Definition 3.1. Let $f$ be a squarefree polynomial of degree 6 over a field $F$. We denote the algebra $F[x] /(f)$ by $A_{f}$. Let $\delta \in A_{f}^{*}$. Fix coordinates $x_{0}, \ldots, x_{5}$ on $\mathbb{P}^{5}(F)$, so that $F\left[x_{0}, \ldots, x_{5}\right]$ is the coordinate ring of $\mathbb{P}^{5}$, and let $g$ be the element $\sum_{i=0}^{5} x^{i} \otimes x_{i} \in A_{f} \otimes_{F} F\left[x_{0}, \ldots, x_{5}\right]$ (we think of $g$ as a "generic element of $A_{f}$ "). We may then uniquely write $g^{2} \delta=\sum_{i=0}^{5} x^{i} \otimes C_{i}$. It is clear that the $C_{i}$ are homogeneous polynomials of degree 2 .

Definition 3.2. Given $f, \delta$ as above, let $V_{f, \delta}$ be the subscheme of $\mathbb{P}^{5}$ defined by $C_{3}, C_{4}, C_{5}$. We call $V_{f, \delta}$ the twist of $V_{f, 1}$ by $\delta$.

Observe that $V_{f, \delta}$ and $V_{f, \delta \alpha^{2}}$ are isomorphic by a linear change of coordinates induced by multiplication by $\alpha$ on $A_{f}$. In particular, if $F$ is algebraically closed, then the isomorphism class of $V_{f, \delta}$ does not depend on $\delta$. 
Theorem 3.3 ([3, Section 16.3]). Let $f$ be as above and let $C$ be the curve of genus 2 defined by $y^{2}=f$. The minimal desingularization of the quotient of $\operatorname{Jac}(C)$ by \pm 1 is isomorphic to $V_{f, 1}$ over $F$.

We now recall some results from [7] on the Picard group of $V_{f, \delta}$. In this section let $F$ be a field of characteristic not equal to 2 , let $f \in F[x]$ be a squarefree polynomial of degree 6 , and let $\delta$ be a unit in $F[x] /(f)$.

Theorem 3.4 ([7, Lemma 2.10, Propositions 2.11 and 2.30]). The variety $V_{f, \delta}$ contains 32 lines. Generically, their classes generate the NéronSeveri group of $V_{f, \delta}$. The lines can be indexed by pairs of complementary subsets $S, \bar{S}$ of $\{1, \ldots, 6\}$ in such a way that $L_{S}$ intersects $L_{S^{\prime}}$ if and only if $S$ or $\bar{S}$ differs from $S^{\prime}$ in exactly one element.

We usually write $L_{S}$ rather than $L_{\{S, \bar{S}\}}$. For example, $L_{1}$ intersects $L_{12}$ but not $L_{24}$ or $L_{123}$. On the other hand, $L_{123}$ does intersect $L_{45}$, because the complement of $\{1,2,3\}$ is $\{4,5,6\}$, which differs from $\{4,5\}$ only in containing 6 .

Definition 3.5. Let $\mathrm{NS}=\mathrm{NS}_{f, \delta}$ be the subgroup of the Néron-Severi group of $V_{f, \delta}$ generated by the classes of lines.

For a description of the Galois action on the 32 lines and on the NéronSeveri group, see [7, in particular Lemma 2.38, Proposition 2.39, and the following discussion.

Definition 3.6. Let $F, f, \delta$ be as above. Let $K$ be the splitting field of $f$ and let $\epsilon_{i}$ be the natural embeddings of $A_{f}$ into $K$. Then $\delta_{i}=\epsilon_{i}(\delta)$.

THEOREM 3.7.

(i) Suppose that $f$ and $\delta$ are generic: in other words, suppose that the Galois group of $f$ is $S_{6}$ and that the extension $F\left(\sqrt{\delta_{i} / \delta_{j}}\right) / F$ has degree 32 . Then $H^{0}(F, \mathrm{NS}) \cong \mathbb{Z}$, generated by a class that intersects every line once.

(ii) Suppose that $f$ is generic and that $\delta$ is generic except for having square norm from $F[x] /(f)$ to $F$. Then there are two orbits $\mathcal{E}, \mathcal{O}$ of lines under the action of Galois and $H^{0}(F, N S) \cong \mathbb{Z} \oplus \mathbb{Z}$.

Proof. The first statement of (ii) is a special case of [7, Lemma 2.37]. In light of this, the statements on Néron-Severi groups are easily verified by computer calculation [6]. The generator in (i) is the hyperplane class of $V_{f, \delta}$.

In the case of $\delta$ having square norm, we will write $i(D)$ for a rational divisor or divisor class $D$ to mean $\left(D \cdot l_{1}, D \cdot l_{2}\right)$, where $l_{1}$ is a line in the orbit $\mathcal{E}$ and $l_{2}$ a line in $\mathcal{O}$. Sometimes we will refer to $i(D)$ as the type of $D$. 
Observe that if $i(D)=(a, b)$ and $\sigma$ is an element of $\operatorname{Gal}(\bar{F} / F)$ that fixes $D$ and exchanges $\mathcal{E}$ and $\mathcal{O}$, then $i\left(D^{\sigma}\right)=(b, a)$.

Definition 3.8. The hyperplane class of [7], which intersects every line once, will be denoted $H$.

Proposition 3.9. Suppose that $N(\delta)$ is a square and that $f$ and $\delta$ are otherwise generic. Then $H^{0}(F, \mathrm{NS})$ is generated by divisors of types $(1,1)$ and $(2,0)$.

Proof. The divisor $H$ is of type $(1,1)$. On the other hand, the divisor $D_{\emptyset}=2 L_{\emptyset}+\sum_{i=1}^{6} L_{i}$ has intersection $-2 \cdot 2+6$ with $L_{\emptyset}$, while its intersection with $L_{i}$ is $2-2=0$. Its intersection with $L_{i j}$ is 2 , because $L_{i j}$ meets $L_{i}$ and $L_{j}$, and it does not meet $L_{i j k}$. Therefore its intersection with a line depends only on whether the line belongs to $\mathcal{E}$ or to $\mathcal{O}$; its type is therefore $(2,0)$. Since the lines generate the Néron-Severi group over $\bar{F}$, the class of this divisor is defined over the field of definition of $\mathcal{E}$. In general, this field of definition is $F(\sqrt{N(\delta)})$, but it is $F$ here because $N(\delta)$ is a square.

To complete the proof, we show that there is no divisor of type $(1,0)$. Indeed, such a divisor would be linearly equivalent to $D_{\emptyset} / 2$. Its self-intersection would be $\left(-2^{2} \cdot 2-6 \cdot 2+2 \cdot 6 \cdot 2\right) / 4=1$. However, the Riemann-Roch theorem for surfaces ([5, Theorem V.1.6]) shows that the self-intersection of every divisor on a $K 3$ surface is even.

We are only interested in $V_{f, \delta}$ that are everywhere locally solvable. This condition implies that every divisor class in $H^{0}(F, \mathrm{NS})$ is represented by an $F$-rational divisor.

Now we consider the Brauer group. Recall from [12, Corollary 2.3.9] that $\operatorname{ker}(\operatorname{Br} V \rightarrow \operatorname{Br} \bar{V}) / \operatorname{im}(\operatorname{Br} F \rightarrow \operatorname{Br} V)$ is isomorphic to $H^{1}(F, \operatorname{Pic} \bar{V})$. Also, the natural inclusion of NS into Pic $\bar{V}$ induces a homomorphism $H^{1}(F, \mathrm{NS})$ $\rightarrow H^{1}(F, \operatorname{Pic} \bar{V})$.

TheOREM 3.10. In the generic case, $H^{1}(F, \mathrm{NS})$ has order 2 . The unique nontrivial element is in the kernel of the restriction map

$$
H^{1}(F, \mathrm{NS}) \rightarrow H^{1}(F(\sqrt{N(\delta)}), \mathrm{NS}) .
$$

Proof. This is a straightforward calculation using Magma [1]. See [6] for details.

Let $F^{\prime}=F(\sqrt{N(\delta)})$. By the inflation-restriction sequence, the nontrivial element of $H^{1}(F, \mathrm{NS})$ is in the image of the inflation map

$$
H^{1}\left(F^{\prime} / F, \mathrm{NS}^{F^{\prime}}\right) \rightarrow H^{1}(F, \mathrm{NS}) .
$$

Since $\operatorname{Gal}\left(F^{\prime} / F\right)$ is cyclic, the group $H^{1}\left(F^{\prime} / F, \mathrm{NS}^{F^{\prime}}\right)$ is identified with $\left(\operatorname{ker} N: \mathrm{NS}^{F^{\prime}} \rightarrow \mathrm{NS}^{F}\right) /\left((\sigma-1) \mathrm{NS}^{F^{\prime}}\right)$, where $\sigma$ generates $\operatorname{Gal}\left(F^{\prime} / F\right)$. If we choose a class $D$ in $\mathrm{NS}^{F^{\prime}}$ representing the nontrivial element of the quotient, 
we see ([13, Lemma 1]) that the nontrivial element of the Brauer group is represented by an Azumaya algebra which is the quaternion algebra $(N(\delta), f)$, where $f$ is a function on $V$ whose divisor is the norm from $F^{\prime}$ to $F$ of a divisor of the class $D$ defined over $F^{\prime}$.

In particular, let $D$ be a divisor on $V \otimes F^{\prime}$ with $i(D)=(1,-1)$. Then $N_{F^{\prime} / F}(D)$ is principal but $D$ is not. Of course, $D$ is not effective, but $D+H$ may be; if we can find an effective divisor $E$ of type $(2,0)$, then $E+E^{\sigma}-2 H=$ $N_{F^{\prime} / F}(E-H)$ is principal. This means that $E-H$ represents the nontrivial element of the quotient as described above. Letting $f_{E}$ be a function whose divisor is $N(E-H)$, we see that the Brauer element is represented by the quaternion algebra $\left(N(\delta), f_{E}\right)$.

In fact, the divisor $E+E^{\sigma}$ is linearly equivalent to twice the hyperplane class on $V_{f, \delta}$, so it is defined by some quadratic form $q \in \mathcal{O}_{\mathbb{P}^{5}}(2)$. (Here we use the fact that $V_{f, \delta}$ is projectively normal, which is true because it is a nonsingular, and hence normal, complete intersection of positive dimension ([5, Exercise 2.8.4(b)]).) The nontrivial Brauer element is then represented by $\left(N(\delta), q / l^{2}\right)$, where $l$ is any nonzero element of $\mathcal{O}_{\mathbb{P}^{5}}(1)$.

Proposition 3.11. Let $Q$ be a quartic surface in $\mathbb{P}^{3}$ such that there is a birational equivalence $\phi: V \rightarrow Q$ that contracts the lines in $\mathcal{O}$ and takes the lines in $\mathcal{E}$ to conics on $Q$. Then the inverse image by $\phi$ of a hyperplane section of $Q$ is a divisor of type $(2,0)$ on $V$. Conversely, the map from $V$ to projective space given by the complete linear system of divisors of type $(2,0)$ has image a quartic surface in $\mathbb{P}^{3}$, takes lines in $\mathcal{E}$ to conics, and contracts lines in $\mathcal{O}$.

Proof. The first statement is clear. For the second, we first note that this linear system has no base points. Indeed, it contains the divisors $D_{1}=$ $2 L_{\emptyset}+\sum L_{i}, D_{2}=2 L_{12}+L_{1}+L_{2}+\sum L_{12 i}$, and $D_{3}=2 L_{34}+L_{3}+L_{4}+\sum L_{34 i}$; no component is shared among these three. Suppose that $P$ is a point on all three of these divisors. Then $P \notin L_{\emptyset}$, because $L_{\emptyset} \cap D_{2}$ is supported on the points of intersection of $L_{1}$ and $L_{2}$ with $L_{\emptyset}$, and these points do not lie on $D_{3}$ because no component of $D_{3}$ meets $L_{1}$ or $L_{2}$. Also, $P \notin L_{1} \cup L_{2}$ for the same reason, and $P \notin \bigcup_{i=3}^{6} L_{i}$ because no component of $D_{2}$ meets any of these lines. We have exhausted all points of $D_{1}$, so this is a contradiction.

It follows that the degree of the image is the self-intersection of a divisor of type $(2,0)$, which in the proof of Proposition 3.9 we showed to be 4 . By Riemann-Roch the dimension of this linear system is 3 , so the image is a surface in $\mathbb{P}^{3}$. Since such a divisor intersects a line in $\mathcal{E}$ twice, the images of lines in $\mathcal{E}$ are curves of degree 2, which are plane conics. Lines in $\mathcal{O}$ are contracted, because these divisors intersect them with degree 0 .

REMARK 3.12. Let $F$ be the field of definition of $V$ and $F^{\prime}$ that of $\mathcal{E}$ and $\mathcal{O}$. The quartic surface $Q$ on which $E$ is a hyperplane section is not 
defined over $F$, but only over $F^{\prime}$. Therefore $E^{\sigma}$ is not a hyperplane section of $Q$, but of $Q^{\sigma}$, and there is no reason to expect that it will be linearly equivalent to $E$. In fact, $Q$ is isomorphic to $Q^{\sigma}$ over $F^{\prime}$, but not by a map induced by a linear automorphism of the ambient $\mathbb{P}^{3}$.

4. The quartic Kummer surface. We now introduce another construction of the Kummer surface. Suppose that $Q_{1}, Q_{2} \in F\left[x_{0}, \ldots, x_{5}\right]$ are quadratic forms such that the scheme $Q_{1}=Q_{2}=0$ is nonsingular and of dimension 3. Let $M_{i}$ be the symmetric matrix corresponding to $Q_{i}$ for $i=1,2$, and let $d(x)=\operatorname{det}\left(M_{1}+x M_{2}\right)$. Our assumption guarantees that $f$ is squarefree and of degree 5 or 6 .

REMARK 4.1. The variety of lines on the scheme $Q_{1}=Q_{2}=0$ is the intersection of the varieties of lines on the schemes $Q_{1}=0$ and $Q_{2}=0$.

Suppose that the scheme $S_{1}$ defined by $Q_{1}=0$ is identified with the Grassmannian $G$ of lines in $\mathbb{P}^{3}$. A line on $S_{1}$ then corresponds to a family of lines in a fixed plane containing a fixed point. The variety of planes on $S_{1}$ has two components: as we saw above, these correspond to families of lines containing a fixed point and families of lines lying in a fixed plane. If we fix a component $P_{i}$ of the variety of planes on $S_{1}$, it is clear that each line on $S_{1}$ is contained in exactly one plane of $P_{i}$. We therefore have a map $\pi_{i}$ from the variety of lines on $Q_{1}=Q_{2}=0$ to the base of $P$, which is isomorphic to $\mathbb{P}^{3}$. The images of $\pi_{1}$ and $\pi_{2}$ are dual to each other and both are isomorphic to the Kummer surface of the Jacobian of the curve $y^{2}=-d(x)$. See [3. Section 17.2] for more details on this.

We now consider the effect of choosing the quadrics $C_{4}, C_{5}$, defined previously as coefficients of $g^{2} \delta$, to be $Q_{1}, Q_{2}$. Let $c_{0}, \ldots, c_{5}$ be the coefficients of $g^{2}$ (with $\delta=1$ ), and let $\iota$ be an automorphism of $\mathbb{P}^{5}$ induced by multiplication by a square root of $\delta$; then $\iota$ induces an isomorphism from the scheme defined by any subset of the $C_{i}$ to the corresponding subset of the $c_{i}$. In particular, $\iota$ induces an isomorphism from the variety of lines of the variety defined by $C_{4}, C_{5}$ to that of the variety defined by $c_{4}, c_{5}$.

Proposition 4.2. The variety of lines on the scheme $C_{4}=C_{5}=0$ is geometrically isomorphic to the Jacobian of $y^{2}=d(x)$. The determinant of the matrix associated to $C_{5}$ is $-N(\delta)$.

Proof. To prove the first statement, we may assume that $\delta=1$, because the base field is algebraically closed. Also, multiplying $d(x)$ by a constant does not change $C_{4}, C_{5}$, or the $\bar{F}$-isomorphism class of the Jacobian of $y^{2}=d(x)$, so we may assume that $d$ is monic. We calculate $C_{4}, C_{5}$, and the associated matrices. Using Magma [1] to evaluate the determinant, we observe that it is equal to $-d\left(-x-a_{5}\right)$, where $a_{5}$ is the $x^{5}$ coefficient of $d(x)$; 
the result then follows from [3, Theorem 17.0.1], since passing to the algebraic closure trivializes the principal homogeneous space. See [6] for details and forthcoming work of van Luijk et al. for a more enlightening proof.

For the second statement, we first show that the determinant of the matrix associated to $c_{5}$ is -1 . The coefficient of $x_{i} x_{j}$ in $c_{5}$ is 0 if $i+j<5$ and 2 if $i+j=5$, so the matrix has 1's along the diagonal from bottom left to top right and 0's above and to the left of this diagonal. Switching row $i$ with row $7-i$ for $i=1,2,3$ changes the sign of the determinant and produces an upper triangular matrix with 1's on the main diagonal; the determinant of such a matrix is 1 . The claim follows.

We obtain the $C_{5}$ associated to $f, \delta$ from that associated to $f, 1$ by a change of base on $\bar{F}[x] /(f(x))$ that divides elements by a square root of $\delta$; by definition of norm, the determinant of this base change is $\sqrt{N(\delta)}$, so the determinant of the matrix is multiplied by $\sqrt{N(\delta)}^{2}=N(\delta)$.

If $C_{5}$ defines a subscheme of $\mathbb{P}^{5}$ isomorphic over $F$ to $G$, then let us fix an $F$-isomorphism $\gamma$. Let $l_{\gamma}$ be the $F$-isomorphism induced by $\gamma$ from the variety of lines on $C_{5}=0$ to that on $G$, and define two maps $\pi_{\delta, i}=\pi_{i} \circ l_{\gamma}$ $\left(i \in\{1,2\}\right.$ ) from the variety of lines on $C_{5}=0$ to $\mathbb{P}^{3}$ (recall the $\pi_{i}$ from Section 2). Let the images of the variety of lines on $C_{4}=C_{5}=0$ under the $\pi_{\delta, i}$ be $K_{\delta, 1}, K_{\delta, 2}$.

Theorem 4.3. Suppose that $C_{5}=0$ defines a subscheme of $\mathbb{P}^{5}$ that is $F$-isomorphic to $G$. Then the minimal desingularizations of the $K_{\delta, i}$ are isomorphic to $V_{f, \delta}$.

Proof. It suffices to prove this for $i=1$. The $\bar{F}$-isomorphism between $V_{f, \delta}$ and $V_{f, 1}$ is the restriction of the automorphism $\iota$ of $\mathbb{P}^{5}$. On the other hand, $\iota$ induces an automorphism of the variety of lines in $\mathbb{P}^{5}$ in an obvious way, and hence an isomorphism between the varieties of lines on $C_{4}=C_{5}=0$ and $c_{4}=c_{5}=0$ (because $\iota$ is an isomorphism from the first of these to the second). Let us call this isomorphism $\kappa$. It is clear that, for all $\sigma$ in $\operatorname{Gal}(\bar{F} / F)$, the isomorphism from $K_{\delta, 1}$ to $K_{1,1}$ induced by $\iota^{\sigma}$ in this way is $\kappa^{\sigma}$. This means that the Galois cocycles expressing $V_{f, \delta}$ and $K_{\delta, 1}$ as forms of $V_{f, 1}$ and $K_{1,1}$ respectively can be identified. From this and the fact that $V_{f, 1}$ and $\tilde{K}_{1,1}$ are isomorphic, it follows that $V_{f, \delta}$ and $\tilde{K}_{\delta, 1}$ are likewise isomorphic.

In view of Propositions 2.3 and 4.2 , the hypothesis can only be satisfied if $N(\delta)$ is a square in $F$. If the norm of $\delta$ is not a square, then the determinant of $C_{5}$ will not be of the form $-r^{2}$, so the two families of planes on $C_{5}$ will not be defined over $F$. In this case it is not possible to define the $\pi_{\delta, i}$ over $F$. This should not be surprising, because the nodes on the image of $\pi_{\delta, i}$ correspond to a set of sixteen disjoint lines on $V_{f, \delta}$, and such a set is only defined when the norm of $\delta$ is a square. 
REMARK 4.4. Alternatively, it is possible that $N(\delta)$ is a square, but nevertheless the variety defined by $C_{5}$ is not $F$-isomorphic to $G$. In this case, Proposition 2.10 shows that no rational planes lie on it, so the twisted maps $\pi_{\delta, i}$ go naturally to a Brauer-Severi variety of dimension 3 with no rational points. In this case the minimal desingularization of the image is still isomorphic to $V_{f, \delta}$. However, this shows that $V_{f, \delta}$ has no rational points; indeed, if $F$ is a number field then $V_{f, \delta}$ must fail to be locally solvable at at least two places.

5. Mapping the intersection of three quadrics to the quartic. Above, we constructed two projective models $V_{f, \delta}, K_{\delta, i}$ of the twisted Kummer surface of the Jacobian of a curve of genus 2. A hyperplane section of $K_{\delta, i}$ misses those -2-curves that are contracted to double points on $K_{\delta, i}$ and intersects twice those that are mapped to conics; that is, it is a divisor of type $(2,0)$. So constructing a divisor of type $(2,0)$, and hence the nontrivial element of the Brauer group of $V_{f, \delta}$, is essentially equivalent to constructing a birational equivalence from $V_{f, \delta}$ to $K_{\delta, i}$. In principle, this could be done using the known equivalence between $V_{f, 1}$ and $K_{1,1}$ ([3, Section 16.3]) and the isomorphisms $\iota$; however, we preferred a different construction, one that avoids all need to trivialize explicit Galois cocycles and almost all need to solve norm equations. (It would be necessary to do an explicit Galois descent over an extension whose Galois group contains $A_{6}$ as a simple factor; that is computationally infeasible.)

We will assume that $F$ is a number field (in fact, our program assumes that $V_{f, \delta}$ is defined over $\mathbb{Q}$ and that $F=\mathbb{Q}(\sqrt{N(\delta)})$; it would be easy to remove this restriction, at the cost of agonizing slowness).

Let us fix $f, \delta$ for the duration of this section. Since we are interested in constructing an example of the Brauer-Manin obstruction in the generic case, we will suppose that $f, \delta$ are generic in the sense of Theorem 3.7. The ideas below should be applicable even when the Galois group is smaller than in the general case, although some reprogramming would be necessary to deal with situations in which there are many Galois orbits of lines.

Given $f, \delta$, we start by calculating the $C_{i}$ defined above. Then we use Proposition 2.3 to determine whether $C_{5}$ defines a scheme isomorphic to the Jacobian. If it does not, then it is not possible to embed $K_{\delta, i}$ in $\mathbb{P}^{3}$ (see Remark 4.4 and in any case $V_{f, \delta}$ is not locally solvable.

If the scheme $C_{5}=0$ is isomorphic to $G$, we will need to find an isomorphism. It suffices to find an $F$-isomorphism of the quadratic forms $C_{5}$ and $Q_{g}=x_{0} x_{5}-x_{1} x_{4}+x_{2} x_{3}$. (This must be possible, because some multiple of $C_{5}$ is isomorphic to $Q_{g}$, while Proposition 2.4 shows that all nonzero multiples of $C_{5}$ are isomorphic as quadratic forms.) The following algorithm, suggested to me by Jonathan Hanke, can be used to find an isomorphism 
of two quadratic forms $Q_{1}, Q_{2}$ in $n$ variables: search for a common value $r$ of the two forms, then write each form as $f_{i}\left(x_{1}, \ldots, x_{n-1}\right)+r x_{n}^{2}$, where the $f_{i}$ are quadratic forms in $n-1$ variables. These forms will be isomorphic, so the problem is solved by induction. (This method can be used to prove Proposition 2.3, see [11, Theorem 9, p. 44].)

However, the program uses a simpler method which is not guaranteed to work. In our situation $C_{5}$ actually has rational coefficients; we diagonalize it over $\mathbb{Q}$ to obtain a diagonal form $D=\sum c_{i} x_{i}^{2}$. If we divide some of the $c_{i}$ by $N(\delta)$ we obtain a form $D^{\prime}$ isomorphic to $D$ over $F$; we can determine whether $D^{\prime}$ is isomorphic to $Q_{g}$ over $\mathbb{Q}$ by calculating the invariants (Proposition 2.3. If it is, we try to construct an isomorphism. First, the product of the coefficients of $D^{\prime}$ is of the form $-n^{2}$. Let $p \mid n$. If $p$ divides all coefficients of $D^{\prime}$, we divide it out. If $p^{2}$ divides the $x_{i}^{2}$ coefficient of $D^{\prime}$, we may remove this factor by replacing $x_{i}$ with $p x_{i}$. If $p$ divides four of the coefficients of $D^{\prime}$, we multiply through by $p$ and then remove the $p^{2}$ as above. Thus we may assume that $n$ is squarefree.

Again, let $p \mid n$, and suppose that $p$ divides the coefficients $d_{1}, d_{2}$ of $x_{1}^{2}, x_{2}^{2}$ in $D^{\prime}$. It is easy to show that the conic $d_{1} x^{2}+d_{2} y^{2}-p d_{1} z^{2}$ fails to be locally solvable at exactly the places where $D^{\prime}$ is not isomorphic to the quadratic form $D^{\prime \prime}=d_{1} x_{1}^{2} / p+d_{2} x_{2}^{2} / p+\sum_{i=3}^{6} d_{i} x_{i}^{2}$. If this conic has a rational point we can construct an isomorphism from $D^{\prime}$ to $D^{\prime \prime}$. (In this case the form $x^{2}+\left(d_{2} / d_{1}\right) y^{2}$ represents $p$, so by standard theory of binary quadratic forms it is equivalent to $x^{2} / p+\left(d_{2} /\left(p d_{1}\right)\right) y^{2}$.) Similarly, if $d_{1} x^{2}+d_{2} y^{2}+p d_{1} z^{2}$ has a rational point then $D^{\prime}$ is isomorphic to $-d_{1} x_{1}^{2} / p-d_{2} x_{2}^{2} / p+\sum_{i=3}^{6} d_{i} x_{i}^{2}$. Repeating this process, we may eventually reach $Q_{g}$.

This method may fail: it is possible that we reach a form for which no primes can be removed from the coefficients in this way. However, experience shows that an element $m$ of $F[x] /(f(x))$ of small norm can usually be found such that multiplying $\delta$ by $m^{2}$ produces $C_{5}$ to which the method applies.

Let $C_{4}^{\prime}$ be $C_{4}$ in the coordinates for which $C_{5}=x_{0} x_{5}-x_{1} x_{4}+x_{2} x_{3}$. We diagonalize $C_{4}^{\prime}$ and find the Fano scheme of lines lying on it. This is a subscheme of $\mathbb{P}^{14}$, and we use the Plücker embedding to find the image. (Diagonalizing $C_{4}^{\prime}$ is not strictly necessary, but it makes the image calculation much faster.) Changing the base of $\mathbb{P}^{5}$ induces a linear change of coordinates on the $\mathbb{P}^{14}$ containing the Grassmannian of lines of $\mathbb{P}^{5}$, so we can recover the subscheme $L$ of $\mathbb{P}^{14}$ parametrizing the lines on $C_{4}^{\prime} \cap G$. The image $\pi_{1}(L)$ is $K_{\delta, 1}$. Since we know that the image is a quartic hypersurface in $\mathbb{P}^{3}$ defined over $F$, we may compute it by applying the maps to the intersection of $L$ with linear subspaces of codimension 2; after the first few images have been computed, the vector space of quartics vanishing on all of the images is 1-dimensional, and so a generator must define the image. 
We could find $K_{\delta, 2}$ by the same method, but this is not necessary for our purposes. If we wished to, it would be simpler to compute the dual of $K_{\delta, 1}$ rather than to calculate $K_{\delta, 2}$ by the same method used for $K_{\delta, 1}$.

Now we turn our attention to $V_{f, \delta}$. We compute the Fano schemes that parametrize the lines on $C_{3}, C_{4}, C_{5}$ and intersect them, producing a scheme of degree 32 and dimension 0 parametrizing the lines of $V_{f, \delta}$. We use elimination ideals to separate this scheme into two components over $F(\sqrt{N(\delta)})$. For each component $B_{i}$ we choose a representative line $M_{i}$. Since $M_{i}$ lies on $C_{4}$ and $C_{5}$, it corresponds to a point $P_{i}$ of $K_{\delta, 1}$. The 32 irreducible curves of self-intersection -2 on $V_{f, \delta}$ correspond to the 16 nodes on $K_{\delta, 1}$ and the 16 tropes. (The tropes are conics on $K_{\delta, 1}$ that are the reduced subschemes of the hyperplane sections of $K_{\delta, 1}$ that pass through 6 nodes. The self-intersection of a generic hyperplane section is 4 , but since these special hyperplane sections are double curves we divide by $2^{2}=4$. Then, as in [8. Section $\mathrm{II}(\mathrm{b})]$, we subtract a correction term of $1 / 2$ for each node.) One of the orbits corresponds to nodes and the other to tropes; by testing which of the $P_{i}$ is singular on $K_{\delta, 1}$, we determine which orbit corresponds to nodes. Let us suppose it is $P_{1}$. Then the lines of $B_{1}$ are defined over the same field as the nodes of $K_{\delta, 1}$, so any map from $V_{f, \delta}$ to $K_{\delta, 1}$ must contract the lines to the nodes.

Since the hyperplane section of $K_{\delta, 1}$ is of type $(2,0)$, the difference $2 H_{V_{f, \delta}}-H_{K_{\delta, 1}}$ is effective (being of type $\left.(0,2)\right)$. Therefore there are quadratic forms on $\mathbb{P}^{5}$ defining the birational equivalence. Furthermore, as we saw above, such maps must contract the lines of $B_{1}$.

Conversely, let $\phi: V_{f, \delta} \rightarrow \mathbb{P}^{3}$ be a map defined by quadratic forms that contracts the lines of $B_{1}$ and whose image is not contained in a hyperplane. Then the base locus must intersect every line of $B_{1}$ twice. Hence either the base locus is a divisor of type $(0,2)$, in which case $\phi$ must be the desired map that embeds $V_{f, \delta}$ by a divisor of type $(2,0)$, or it consists of two points on each line of $B_{1}$, for a total of 32 points.

Proposition 5.1. With $\phi$ as above, the base locus of $\phi$ is in fact a divisor of type $(0,2)$, not two points on each line of $B_{1}$.

Proof. Since $V_{f, \delta}$ is of degree 8, the self-intersection of the zero locus of a quadratic form is $8 \cdot 2^{2}=32$. Each base point reduces the degree of the image by 1 , so if the map had 32 base points the degree of the image would be 0 ; that is, the image would be of lower dimension.

If the dimension of the image were 0 , then the image would be contained in a hyperplane; so suppose it is 1 . The image is then an irreducible curve $X$ that contains all sixteen nodes of $K_{\delta, 1}$. Since the lines of $B_{2}$ are not contracted, they must map surjectively to $X$, which is therefore the image of a 
line by a map defined by quadratic forms and whose degree is therefore at most 4 .

Let $T$ be a trope, let $\tilde{K}_{\delta, 1}$ be the minimal desingularization of $K_{\delta_{1}}$, and let $N_{1}, \ldots, N_{6}$ be the exceptional divisors above the nodes lying on $T$. On $\tilde{K}_{\delta, 1}$, then, we have $\tilde{H} \sim 2 \tilde{T}+\sum N_{i}$. Intersecting both sides with the strict transform $\tilde{X}$ of $X$, we see that $\tilde{H} \cdot \tilde{X} \leq 4$ and $\tilde{X} \cdot N_{i} \geq 1$ for all $i$; it follows that $\tilde{X} \cdot \tilde{T}<0$. Since $X$ and $T$ are irreducible curves, this means that $X=T$. But $X$ cannot be a trope, because the tropes do not contain all sixteen nodes. This contradiction establishes that $\phi$ is the desired map.

So we find all 4-tuples of quadratic forms defined over $F(\sqrt{N(\delta)})$ that contract the lines of $B_{1}$ to a node of $K_{\delta, 1}$ and that do not define a constant map. (We know that such maps exist, so this amounts to finding all elements of a vector space not contained in a certain proper subspace; this is simple linear algebra.) Once we have found one such map, corresponding to a divisor $D_{0}$ of type $(0,2)$, we may find other equations for it. To do so, find a divisor of type $(2,2)$ containing $D_{0}$ (this is just the divisor cut out by a quadratic form vanishing on $\left.D_{0}\right)$; the residual is then of type $(2,0)$. Every effective divisor of type $(0,2)$ is then the residual intersection of $V_{f, \delta}$ with a quadratic form vanishing on this divisor.

To describe the Brauer group, let us pull back hyperplanes $H_{1}, \ldots, H_{n}$ from $K_{\delta, 1}$ to $V_{f, \delta}$ and let $q_{i}(1 \leq i \leq n)$ be the quadratic forms defining the union of the inverse images with their $K(\sqrt{N(\delta)})$-conjugates as subschemes of $V_{f, \delta}$; then the $\left(N(\delta), q_{i} / l_{i}^{2}\right)$ are Azumaya algebras representing the nontrivial element of $\operatorname{Br} V_{f, \delta} / \mathrm{Br} \mathbb{Q}$, where $l_{i}$ are arbitrary nonzero linear forms. The construction does not guarantee that these Azumaya algebras represent the same element of $\operatorname{Br} V_{f, \delta}$.

6. Example of Brauer-Manin obstruction. In this section we give an example of $f$ and $\delta$ such that the Brauer-Manin obstruction blocks the existence of rational points on the Kummer surface of the Jacobian of $y^{2}=$ $f(x)$ twisted by $\delta$. The reader unfamiliar with the Brauer-Manin obstruction may wish to consult [4, Section 2].

The following simple lemma will allow us to compute local invariants of an element of the Brauer group at all but finitely many places.

LEMMA 6.1. Let $p>40$ be an odd prime and $n$ an integer relatively prime to $p$. Let $S$ be a smooth $K 3$ surface over $\mathbb{Q}$ with good reduction at $p$ in $\mathbb{P}^{5}$ defined by three quadratic forms, and $f$ a quadratic form over $\mathbb{Q}_{p}$ that is not identically 0 on $S$, is not $0 \bmod p$, and does not have any coefficients with $p$ in the denominator. Then there is a $\mathbb{Q}_{p}$-point $P_{p}$ of $S$ such that the local invariant $\left(n, f\left(P_{p}\right)\right)_{p}$ is 1 . 
REMARK 6.2. Of course, $f\left(P_{p}\right)$ is not well-defined, but it is defined up to multiplication by an element of $\left(\mathbb{Q}_{p}^{*}\right)^{2}$, so the local invariant is well-defined.

Proof of Lemma 6.1. The Weil bounds show that $S$ has at least $p^{2}-22 p+1$ points $\bmod p$. The locus of $S \bmod p$ where $f=0(\bmod p)$ is a curve of degree 16 over $\mathbb{F}_{p}$, so it has at most $16 p+16$ points $\bmod p$ (with equality if it is a disjoint union of 16 rational lines). Since $p>40$, it follows that there are points of $S \bmod p$ where $f \neq 0$. Let $P$ be such a point of $S \bmod p$. Since $S$ has good reduction at $p$, we can lift it to a $\mathbb{Q}_{p}$-point $P_{p}$. Then the $p$-adic valuation of $f\left(P_{p}\right)$ is 0 (scaling so that the coordinates of $P_{p}$ are in $\mathbb{Z}_{p}$ and not all in $\left.p \mathbb{Z}_{p}\right)$. Since $p$ is odd and does not divide $n$, it follows that $\left(n, f\left(P_{p}\right)\right)_{p}=1$.

We now proceed to the proof of our main arithmetical theorem.

Theorem 6.3. Let $f=x^{6}-3 x^{5}+3 x^{4}-3 x^{2}-x-1$, let $A_{f}=\mathbb{Q}[x] /(f(x))$, and let $\delta \in A_{f}$ be the image of $x^{5}-7 x^{4}+4 x^{3}+3 x^{2}+x$. Then the BrauerManin obstruction blocks the existence of rational points on the surface $V_{f, \delta}$, which is defined by the equations

$$
\begin{aligned}
3 x_{1}^{2} & +3 x_{1} x_{2}-4 x_{1} x_{3}-19 x_{1} x_{4}-26 x_{1} x_{5}-7 x_{1} x_{6}-2 x_{2}^{2}-19 x_{2} x_{3} \\
& -26 x_{2} x_{4}-7 x_{2} x_{5}+54 x_{2} x_{6}-13 x_{3}^{2}-7 x_{3} x_{4}+54 x_{3} x_{5}+125 x_{3} x_{6} \\
& +27 x_{4}^{2}+125 x_{4} x_{5}+112 x_{4} x_{6}+56 x_{5}^{2}-105 x_{5} x_{6}-261 x_{6}^{2}, \\
x_{1}^{2} & -8 x_{1} x_{3}-8 x_{1} x_{4}+10 x_{1} x_{5}+62 x_{1} x_{6}-4 x_{2}^{2}-8 x_{2} x_{3}+10 x_{2} x_{4} \\
& +62 x_{2} x_{5}+134 x_{2} x_{6}+5 x_{3}^{2}+62 x_{3} x_{4}+134 x_{3} x_{5}+184 x_{3} x_{6} \\
& +67 x_{4}^{2}+184 x_{4} x_{5}+164 x_{4} x_{6}+82 x_{5}^{2}+128 x_{5} x_{6}+183 x_{6}^{2}, \\
5 x_{1}^{2} & +17 x_{1} x_{2}+34 x_{1} x_{3}+3 x_{1} x_{4}-74 x_{1} x_{5}-191 x_{1} x_{6}+17 x_{2}^{2}+3 x_{2} x_{3} \\
& -74 x_{2} x_{4}-191 x_{2} x_{5}-222 x_{2} x_{6}-37 x_{3}^{2}-191 x_{3} x_{4}-222 x_{3} x_{5} \\
& -33 x_{3} x_{6}-111 x_{4}^{2}-33 x_{4} x_{5}+382 x_{4} x_{6}+191 x_{5}^{2}+601 x_{5} x_{6}-137 x_{6}^{2} .
\end{aligned}
$$

Proof. The norm of $\delta$ is $123008=2^{7} 31^{2}$, so the nontrivial Brauer element is of the form $(2, f)$. The only primes ramified in $A_{f}$ are 2 and 14009 , so the only primes of bad reduction of $V_{f, \delta}$ are 2,31 , and 14009 . Since 2 is a square in $\mathbb{Q}_{31}, \mathbb{Q}_{14009}$, and $\mathbb{Q}_{\infty}$, the local invariants at these places are identically 0.

Using the programs, we find an explicit Brauer element $\left(2, f_{i} / l^{2}\right)$, where the $f_{i}$ are quadratic polynomials. These are relegated to [6] because the smallest of them, which we will call $f_{1}$, has coefficients with up to 19 digits.

Let $P_{b}=\{2,31,14009\}$ be the set of primes of bad reduction of $V$ and let $P_{s}$ be the set of primes less than 23 . To show that $V$ is locally solvable at all finite places, it is enough to prove that it has a point in $\mathbb{Q}_{p}$ for all $p \in P_{b} \cup P_{s}$, since for other $p$ there is automatically a smooth point $\bmod p$ by the Weil bounds and such a point lifts to $\mathbb{Q}_{p}$ by Hensel's lemma. It is easy to find 
smooth points mod all odd primes less than 23 and mod 31, and there is a smooth point mod 14009 with $x_{3}=x_{6}=0$. On the other hand, $V$ has no smooth points mod 2 , but there is a 2 -adic point with $x_{3}-3 x_{6}=x_{5}=0$. In addition, there is a real point with $x_{5}=x_{6}=0$.

It follows from Theorem 1 of [2] that the local invariant is constant at primes of good reduction; since 2 is a square mod 31 and mod 14009, the local invariant is identically 0 there. To prove the theorem, it therefore remains to verify the following two claims:

(i) the local invariant of the nontrivial Brauer element at 2 is constant;

(ii) $\sum_{p}\left(2, b\left(P_{p}\right)\right)=1 / 2$.

These claims are easily checked by computation. To prove claim (i), the program begins by calculating the invariants of $\left(2, f_{i}\right)$ at a single 2 -adic point for all $i$ to determine the class of $\left(2, f_{1}\right)-\left(2, f_{i}\right)$ in $\operatorname{Br} \mathbb{Q}_{2}$. It then finds all of the points of $V \bmod 2$ and creates a polydisc with all radii $1 / 2$ around each point; every $\mathbb{Q}_{2}$-point of $V$ must be contained in one of these. For each polydisc, the program attempts to use the $f_{i}$ to prove that the local invariant is constant there. If it is, and the invariant is not already known to occur, the program then tries to prove that no 2-adic points of $V$ are found in the polydisc. If not, the program subdivides the polydisc and proceeds to examine the subdivisions. (This is much the same method discussed in the proof of [7, Theorem 3.31], except that we use depth-first rather than breadth-first search; it is much faster to manipulate the end of a long list rather than the beginning, and that is what depth-first search does.) The program expands about 40000 polydiscs, in some cases working mod $2^{10}$, before concluding that the local invariant at 2 is identically $1 / 2$.

Lemma 6.1 reduces claim (ii) to a finite calculation. The primes dividing the denominator of some coefficient of $f_{1}$ are 11, 59, 113, 1531, 20161; the program finds that $\left(2, b\left(P_{p}\right)\right)$ is equal to $1 / 2$ for $p=2,11,1531$ and to 0 for the other primes less than 40 and for 59, 113, 20161. Finally, since 2 is a square in $\mathbb{R}$ the invariant is 0 there.

REMARK 6.4. The pair $(f, \delta)$ above was found by searching through a table of extensions of $\mathbb{Q}$ of degree 6 for fields with an element $\nu$ of norm 2 such that the local invariant of the twist of the $K$, Kummer surface of the Jacobian of $y^{2}=f(x)$, by $\nu$ is constant at all $p$ (the only $p$ that need to be considered are 2 and the ramified primes of $\left.A_{f}=\mathbb{Q}[x] /(f(x))\right)$. To simplify matters, one can restrict to the fields that are ramified only at 2 and primes congruent to $\pm 1 \bmod 8$, since the local invariant at $p$ must be 0 if $p \equiv 1$ $(\bmod 8)$. Thus I found the $f$ used above and $\delta_{0}=\frac{1}{2}\left(-x^{4}+x^{3}-x-1\right)$. For this pair $\left(f, \delta_{0}\right)$, the local invariant is constant at all places, but unfortunately the sum is 0 , so there is no Brauer-Manin obstruction. However, multiplying by an element $\beta$ of $A_{f}$ that has square norm and is a square multiplied by an 
integer everywhere locally at 2 will not affect the invariants except at primes involved in $\beta$; moreover, if a prime involved in $\beta$ is congruent to $\pm 1 \bmod 8$, then the local invariant there is automatically 0 . Trying some suitable $\beta$, I found that multiplying $\delta_{0}$ by the element $\frac{1}{2}\left(-x^{5}+5 x^{3}-3 x^{2}-3\right)$ of $A_{f}$ of norm $31^{2}$ made the sum of local invariants $1 / 2$. The given $\delta$ is obtained from this by multiplying by -2 , which does not affect the surface.

In this way, it is easy to find infinitely many $\beta$ up to elements of $\left(A_{f}^{*}\right)^{2} \mathbb{Q}^{*}$ such that the invariants of the surface associated to $\left(f, \delta_{0} \beta\right)$ are all locally constant, and one would expect that half of them (in some sense) give twists of the Kummer surface on which the local invariants sum to 0 , and half $1 / 2$. Accordingly, the following conjecture seems reasonable.

Conjecture 6.5. Up to elements of $\left(A_{f}^{*}\right)^{2} \mathbb{Q}^{*}$, there are infinitely many $\beta$ for which the Brauer-Manin obstruction blocks the existence of rational points on the twist of $K$ by $\beta$.

Acknowledgements. I would like to thank Victor Flynn, Jonathan Hanke, and Ronald van Luijk for helpful discussions of the questions considered in this article. In addition, I am grateful to the Department of Pure Mathematics at the University of Waterloo for its financial support and hospitality during the preparation of this paper and especially to Michael Rubinstein and David McKinnon for arranging for my stay there. I also thank the referee for detailed comments that led to many improvements in the exposition.

\section{References}

[1] W. Bosma, J. Cannon, and C. Playoust, The Magma algebra system. I. The user language, J. Symbolic Comput. 24 (1997), 235-265.

[2] N. Bruin, The arithmetic of Prym varieties in genus 3, arXiv:math/0408069 [math.NT].

[3] J. W. S. Cassels and E. V. Flynn, Prolegomena to a Middlebrow Arithmetic of Curves of Genus 2, London Math. Soc. Lecture Note Ser. 230, Cambridge Univ. Press, 1996.

[4] P. Corn, Tate-Shafarevich groups and K3 surfaces, Math. Comp. 79 (2010), 563-581.

[5] R. Hartshorne, Algebraic Geometry, Grad. Texts in Math. 52, Springer, 1977.

[6] A. Logan, Programs and calculations, available for download from http://www. math.uwaterloo.ca/ a5logan/math/kp.tar.gz.

[7] A. Logan and R. van Luijk, Nontrivial elements of Sha explained through K3 surfaces, Math. Comp. 78 (2009), 441-483.

[8] D. Mumford, The topology of normal singularities of an algebraic surface and a criterion for simplicity, Inst. Hautes Études Sci. Publ. Math. 9 (1961), 5-22.

[9] O. T. O'Meara, Introduction to Quadratic Forms, Springer, 1971. 
[10] M. Reid, The complete intersection of two or more quadrics, $\mathrm{PhD}$ thesis, Cambridge Univ., 1972.

[11] J.-P. Serre, A Course in Arithmetic, Grad. Texts in Math. 7, Springer, 1973.

[12] A. Skorobogatov, Torsors and Rational Points, Cambridge Tracts in Math. 144, Cambridge Univ. Press, 2001.

[13] H. P. F. Swinnerton-Dyer, Brauer-Manin obstructions on some Del Pezzo surfaces, Math. Proc. Cambridge Philos. Soc. 125 (1999), 193-198.

\author{
Adam Logan \\ Centre de Recherches Mathématiques \\ Université de Montréal \\ Case postale 6128, Succursale Centre-ville \\ Montréal, QC, H3C 3J7, Canada \\ E-mail: adam.m.logan@gmail.com
}

Received on 28.2.2008

and in revised form on 9.10.2009 